\title{
Something Old, Something New, Something Borrowed... Comparative Effectiveness Research: A Policy Perspective
}

\author{
Prasun Subedi, PhD; Eleanor M. Perfetto, PhD; and Riaz Ali, BA, MPP
}

$\mathrm{I}$ $\mathrm{n}$ the nearly 18 months that have elapsed following enactment of the Patient Protection and Affordable Care Act (PPACA), health care scholars have debated a key portion of the legislation that has the potential to dramatically impact health care. Section 6301 of the PPACA outlines how the federal government will play an increasingly direct role in shaping comparative effectiveness research (CER). ${ }^{1}$ The inclusion of CER in the PPACA marked a critical step in the advancement of health services research, given that the legislation called for significant federal investment in CER with the ultimate goal of improving the efficiency of the health care system. Here, we briefly review the policy history of CER as it relates to prior efforts in health services research and health technology assessment, discuss the promises and fears associated with CER as outlined in the PPACA, and highlight the important role that the new Patient-Centered Outcomes Research Institute (PCORI) will likely play in advancing both CER methods and evidence generation.

\section{Historical Policy Context}

It is important to recognize that current efforts around CER represent the latest in a series of evolutionary steps that were borne out of the constructs of "health technology assessment" (HTA), "effectiveness research," and "evidence-based medicine," among others. Conceptually, each of these constructs serves a distinct purpose (e.g., evidence generation and analysis versus application in decision making), and was advanced toward discrete objectives (e.g., understanding if something works versus is something worth doing or paying for). ${ }^{2}$ These differences belie a common thread-integration of clinical evidence about an intervention or service into decision-making.

Figure 1 demonstrates a chronology of this activity—dating back several decades_-demonstrating the overall effort of infusing evidence into health care decisions. In the United States, these early activities can be traced back to the Congressional Office of Technology Assessment (OTA, 1972-1995), an agency tasked with oversight of various scientific and technical issues. ${ }^{3}$ Over 2 decades, the OTA issued a series of reports on a variety of process- and disease-oriented health care matters. Although defunded in the mid-1990s as part of government consolidation reforms, the reviews conducted by the OTA set an example for similar processes that have subsequently been developed and implemented by both public and private entities in the United States and other countries. Prior efforts toward evidence integration conducted in the United States have proven useful in broadening knowledge, but federally-funded agencies have faced significant challenges in sustaining their efforts, largely due to political opposition borne out of perceptions that this work was primarily focused on reducing costs, and that its implementation would lead to rationing of health care. ${ }^{4}$

In light of a legacy of challenges and lingering conflation of these distinct concepts, the federal government has again sought to wade carefully_but concertedly-in this space. Recently it has focused its efforts on organizing investment on clinical comparative effectiveness. Table 1 includes a selection of recent definitions of CER advanced by the Agency for Healthcare Research and Quality (AHRQ) which runs the Effective Healthcare Program; the Federal Coordinating Council, which offered a definition to help orient the $\$ 1.1$ billion in funding through the American Recovery and Reinvestment Act; the Institute of Medicine; and the definition of CER provided in the PPACA. These definitions share several common elements: (a) focus on clinical effectiveness; (b) inform a wide range of decision-makers (i.e., patients, providers, and policymakers); and (c) focus on a broad set of interventions and services. More recently, the PCORI released a draft definition of patient-centered outcomes research (PCOR) for public comment. ${ }^{5}$ While the PCOR definition is similar to many of the CER definitions discussed above, its clear focus on preferences and needs marks an important shift towards the patient—and this shift may have important implications for evidence generation and dissemination.

Given that CER-like efforts have been percolating in the United States for the better part of 4 decades, an inevitable question arises: why was CER so clearly singled out as a critical component of the 2010 health care reform legislation? One factor is an acute awareness of the rising costs in health care and growing questions about whether those have translated to meaningful improvement in quality of care. As an example, recent technological advancements have brought about many novel diagnostic and treatment paradigms, but every incremental innovation seemingly brings with it additional questions regarding how the new technology can best be used in the context of existing treatments to improve outcomes (i.e., advancing the quality of care), while not overburdening increasingly constrained resources (e.g., without significantly increasing the cost of care). The majority of the prior CER-like efforts were designed to answer similar questions about quality and cost; however, the PPACA's clear focus on this research is also motivated by the fact that "in the next decade, the United States must absorb 32 million currently uninsured people into the health care system, while simultaneously improving the quality of care and slowing cost increases." ${ }^{\circ}$ While there is significant theoretical promise that comparative studies will provide evidence both to improve quality and to decrease cost, there are also many potential fears regarding the inappropriate 
FIGURE 1 Timeline of Selected Health Technology Assessment, Effectiveness Research, and Evidence-Based Medicine Activities

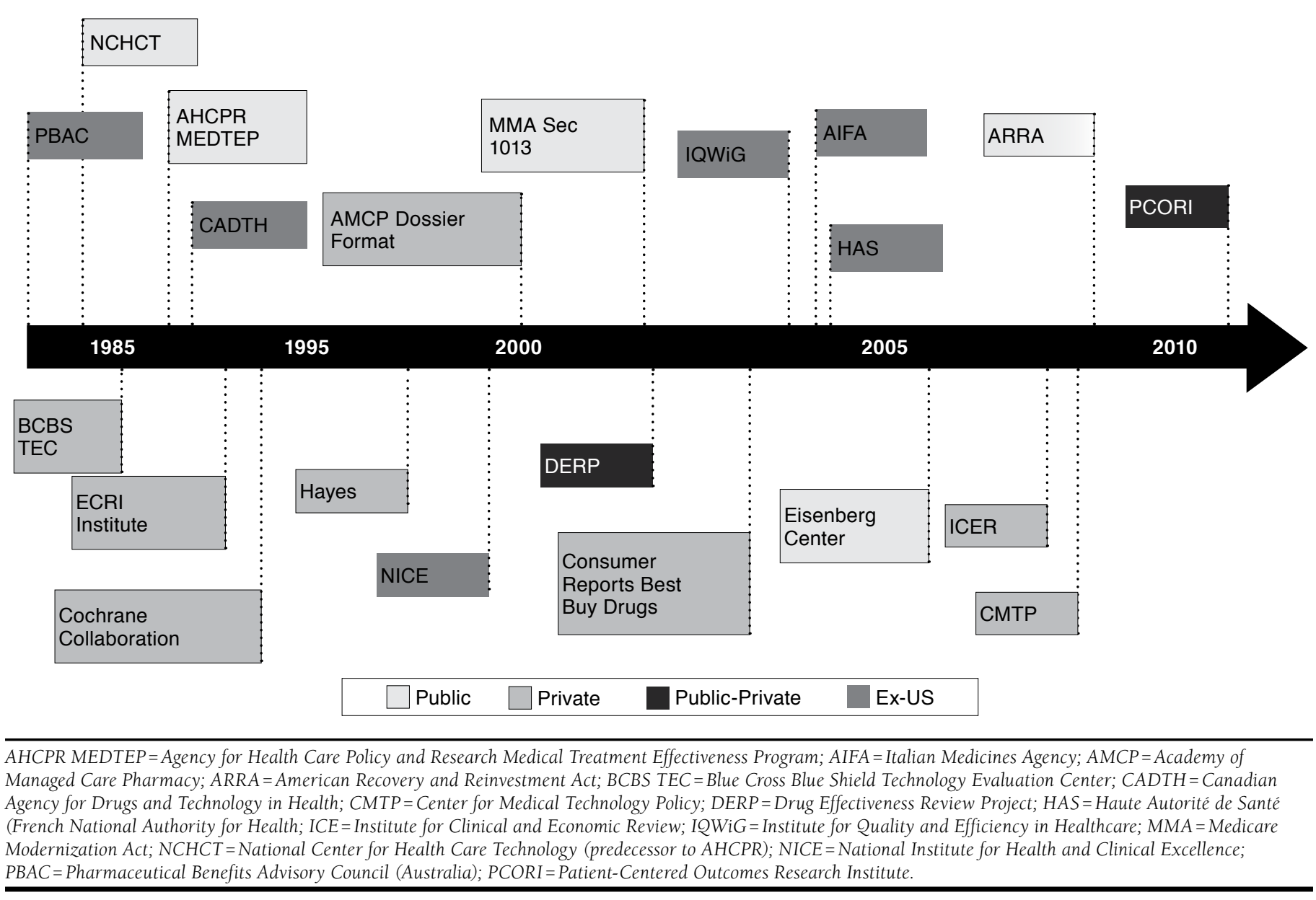

use of CER in the context of financing decision-making.

\section{The Promise and Fear of CER}

The real "promise" of CER lies in its potential to generate "more and better evidence on what works best." There have been significant technological advances in recent years, yet the evidence of effectiveness of health care interventions as a whole is suboptimal. This is partly because much of the published evidence on health care interventions is defined and driven by randomized controlled trials (RCTs) designed to answer specific regulatory questions. Although RCTs are praised for their capacity to identify causal relationships between treatments and health outcomes, it is important to note that these studies are not designed to answer more intricate questions regarding how a new therapy should be considered for use in the context of existing treatment options. RCTs are typically conducted in carefully selected patient populations, under highly controlled settings, with placebo comparators and often provide only aggregated averaged results-largely ignoring variation in treatment response by patient characteristics. By definition, CER studies, with their objective of "comparing health outcomes and the clinical effectiveness of 2 or more medical treatments, services, or items," have the potential to significantly add to the evidence base used in the treatment selection process. In the short term, this evidence is likely to be derived from observational studies conducted using the myriad real-world data sources developed in recent years; in the longer term, it may be that RCT designs are increasingly adapted to provide more relevant evidence for comparative questions.

CER studies that generate evidence through an evaluation of the spectrum of health care interventions and services, that reflect true patient choices for a given clinical situation, will improve patient and physician decision making. With the appropriate evidence, CER has the potential to lead to 


\section{TABLE 1 Select Definitions of Comparative Effectiveness Research}

Source

Agency for Healthcare Research and Quality $(\mathrm{AHRQ})^{\mathrm{a}}$

Federal Coordinating Council (FCC)b

Definition

"Comparative effectiveness research is designed to inform health-care decisions by providing evidence on the effectiveness, benefits, and harms of different treatment options. The evidence is generated from research studies that compare drugs, medical devices, tests, surgeries, or ways to deliver health care."

"The conduct and synthesis of systematic research comparing different interventions and strategies to prevent, diagnose, treat and monitor health conditions. The purpose of this research is to inform patients, providers, and decision-makers, responding to their expressed needs, about which interventions are most effective for which patients under specific circumstances. To provide this information, comparative effectiveness research must assess a comprehensive array of health-related outcomes for diverse patient populations. Defined interventions compared may include medications, procedures, medical and assistive devices and technologies, behavioral change strategies, and delivery system interventions. This research necessitates the development, expansion, and use of a variety of data sources and methods to assess comparative effectiveness."

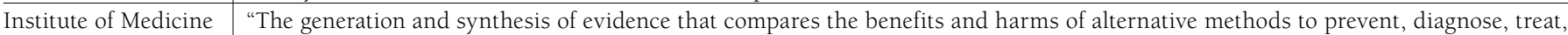
$(\mathrm{IOM})^{\mathrm{c}}$ and monitor clinical conditions, or to improve the delivery of care. The purpose of CER is to assist consumers, clinicians, purchasers, and policy makers to make informed decisions that will improve health care at both the individual and population levels."

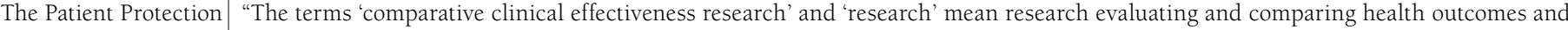
and Affordable Care Act of 2010 (PPACA)d the clinical effectiveness, risks, and benefits of 2 or more medical treatments, services, and items..."

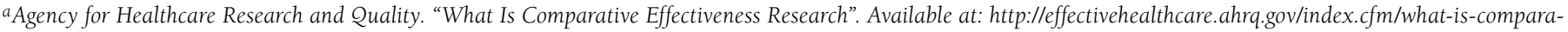
tive-effectiveness-research1/. Accessed September 26, 2011.

${ }^{b}$ Federal Coordinating Council for Comparative Effectiveness Research. Report to the President and Congress. 30 June 2009. Available at: http://www.hhs.gov/recovery/ programs/cer/cerannualrpt.pdf. Accessed September 26, 2011

'IOM (Institute of Medicine). 2009. Initial National Priorities for Comparative Effectiveness Research. Washington, DC: The National Academies Press. Available at:

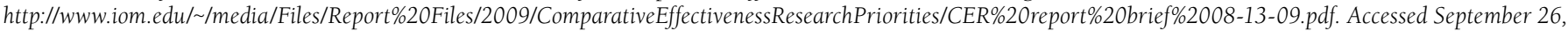
2011.

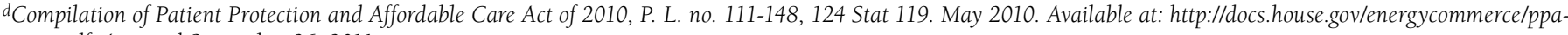
cacon.pdf. Accessed September 26, 2011.

better, more patient-relevant treatment decisions-allowing the "right" treatment to be delivered to the "right" patient in the "right" setting. Achieving this alignment would likely yield significant downstream effects. Specifically, CER evidence, if rigorously produced and effectively transmitted, represents a significant opportunity to reduce current variations in the quality of care, which in turn would serve to improve outcomes and reduce currently observed health care disparities. ${ }^{8}$ Ultimately, CER evidence also has the potential to represent an important step forward in the progression of personalized medicine - the development of "treatment regimens based on the molecular biology of individuals or their diseases"9-which to date has been a promising, albeit elusive, goal.

While the promises of CER are great, so are the "fears" regarding the impact such research may have on how health care is practiced and financed-both for the public and private health insurance markets. In the months leading up to the passage of PPACA, there were significant concerns that government-sponsored health care studies would ultimately lead to homogenized (i.e., "one-size fits all") treatment recommendations that ignored patient heterogeneity. ${ }^{10}$ These anxieties were coupled with worries that such treatment recommendations would inevitably be used to justify cost control efforts, leading to indiscriminate coverage restrictions that could potentially devastating impacts on patients themselves (e.g., "death panels"). ${ }^{11}$ Moreover, some have argued that such blunt restrictions would force a reduction of investment in health care innova- tion, which has advanced clinical paradigms and generated economic value in the United States for many years. ${ }^{12}$

In the end, many of these fears seem to have been heard by Congressional officials and were addressed in the final language of the health care reform legislation. PPACA established the PCORI, and the legislation directed that the public-private institute should seek to "advance the quality and relevance of evidence concerning the manner in which diseases, disorders, and other health conditions can effectively and appropriately be prevented, diagnosed, treated, monitored, and managed," to inform "patients, clinicians, purchasers and policy makers in making informed health decisions." This evidence- and stakeholder-focused language, taken in the context of the PPACA's mandate that the PCORI board (a) establish a expert advisory panel on rare diseases, and (b) provide "support and resources to help patient and consumer representatives effectively participate" in its activities, suggests a clear sensitivity to the fears discussed above. ${ }^{1}$

In addition, the legislation mandates that PCORI not make clinical, coverage, or reimbursement recommendations on the basis of the evidence generated at its direction. The Secretary of Health and Human Services is granted the authority under the establishing legislation to use CER findings from PCORIsponsored work, in conjunction with other evidence, in coverage determinations, but there are specific caveats that coverage decisions based on CER must be developed in a transparent and iterative manner (where iterative refers to the public 
review and peer review processes that must be employed by the Secretary in assessing and determining coverage recommendations), and must not differentiate the value of life for an elderly, disabled, or terminally ill patient relative to a healthy patient. ${ }^{1}$ The Secretary is also forbidden from using a qualityadjusted life year (QALY) to set a threshold for decision making. However, it is expected that private payers will use PCORI findings in their own economic evaluations and will make coverage and reimbursement determinations based on these assessments.

\section{Role of PCORI in Advancing CER Infrastructure and Methods}

A critical aspect of PCORI's remit, especially in the short run, will focus on developing both the infrastructure to support substantive CER studies, as well as the methodological standards by which the research it funds should be carried out. Significant initial investments in these 2 areas have already been made as part of the CER funding allocated through the American Reinvestment and Recovery Act. ${ }^{13}$ From an infrastructure perspective, much work is needed to advance health information technology (HIT) from its current disaggregated state to a point where data sources such as electronic medical records, clinical and claims databases, and patient registries can be appropriately combined for use in CER. PCORI can and likely will play a critical role in ensuring that these and other data from routine, clinical encounters can be appropriately utilized in conjunction with clinical trials (randomized and pragmatic) to serve as a rich source of raw data. Alemayehu and Mardekian provide additional thoughts on the infrastructure requirements needed for secondary data sources to be optimally utilized for CER in a separate article in this supplement (pages S16-S21).

As these data sources are developed, it will be equally important for PCORI to establish a clear methodological framework so that the CER studies it funds can have maximum scientific validity and broad acceptance by the end-user(s). Toward this end, the legislation requires PCORI to establish a Methods Committee, which will seek the advice of experts in biostatistics, health services research, and epidemiologists (among other disciplines) to advise and assist PCORI on developing best practices for conducting CER. ${ }^{1}$ As previously discussed above, a variety of organizations and entities have focused on CER-like efforts in the past. As a result, there exists a fairly substantial foundation of methods and standards from which PCORI can begin its work. However, it is critical that the PCORI board have adequate expert advice with which to understand, interpret, and potentially adopt existing methods standards, as well as develop new guidance. As a potential first step in this process, several articles in this supplement may provide PCORI and other interested stakeholders with "food for thought" in terms of methods development. Alemayehu et al. provide their insights on statistical issues related to the anal- ysis of nonrandomized studies (pages S22-S26); Sanchez et al outline how hybrid studies may be used to answer important CER questions (pages S34-S37); and Alemayehu et al. provide a framework that outlines how patient-reported outcomes may be optimally included in CER studies (pages S27-S33).

\section{Conclusions}

Although the core concepts behind CER have been in development under different labels for a number of years, it is clear that the health care reform effort, as outlined in PPACA, has raised the awareness of CER to demonstrably higher levels. The formation of PCORI, and its clear opportunity to advance both the infrastructure and methods of CER, demonstrates that the federal government recognizes the important role CER can play in addressing both quality and cost considerations. Through clear and open dialogue and engagement with all stakeholders-including patients, providers, insurers, academics, and industry-PCORI can leverage the wealth of existing resources to build an initial framework from which it can advance and promote the appropriate use of CER to improve upon the overall value of health care in the United States.

\section{Authors}

PRASUN SUBEDI, PhD, is Director, Worldwide Policy, and ELEANOR M. PERFETTO, PhD, is Senior Director, Reimbursement and Regulatory Affairs, Federal Government Relations, Pfizer, Inc., New York, New York. RIAZ ALI, BA, MPP, is Director, Avalere Health, LLC, Washington, DC.

\section{DISCLOSURES}

This supplement was funded by Pfizer, Inc. Subedi and Perfetto are Pfizer employees. Ali is an employee of Avalere Health, which receives consulting income from Pfizer and other health care organizations. Ali and Avalere Health did not receive specific consulting fees from Pfizer for Ali's contributions to this manuscript.

Subedi and Perfetto conceived and designed the article, with the assistance of Ali. All 3 authors contributed to writing and revision of the article.

\section{REFERENCES}

1. Compilation of Patient Protection and Affordable Care Act, P. L. no. 111148, 124 Stat 119. May 2010. Available at: http://docs.house.gov/energycommerce/ppacacon.pdf. Accessed September 28, 2011.

2. Luce B, Drummond M, Jönsson B, et al. EBM, HTA, and CER: Clearing the Confusion. Milbank Q. 2010;88(2):277-81

3. Power E, Tunis S, Wagner J. Technology assessment and public health. Annu Rev Public Health. 1994;15:561-79.

4. Luce B, Cohen RS. Health technology assessment in the United States. Int J Technol Assess Health Care. 2009;25(Suppl 1):33-41. 
5. Patient-Centered Outcomes Research Institute. Patient-Centered Outcomes Research Institute asks public for input on definition of 'patientcentered outcomes research. July 20, 2011. Available at: http://www.pcori. org/2011/patient-centered-outcomes-research-institute-asks-public-forinput-on-definition-of-\%E2\%80\%98patient-centered-outcomes-research/. Accessed September 28, 2011.

6. Sox H. Comparative effectiveness research: a progress report. Ann Intern Med. 2010;153(7):469-72.

7. Tunis SR, Benner J, McClellan M. Comparative effectiveness research: policy context, methods development, and research infrastructure. Stat Med. 2010;29(19):1963-76.

8. Mullins CD, Onukwugha E, Cooke JL, Hussain A, Baquet CR. The potential impact of comparative effectiveness research on the health of minority populations. Health Aff (Millwood). 2010;29(11):2098-104.
9. Epstein R, Teagarden JR. Comparative effectiveness research and personalized medicine: catalyzing or colliding? Pharmacoeconomics. 2010;28(10):905-13.

10. Reichard J. 'PCORI' backers eye PR strategy to cool 'death panel' rhetoric. Commonwealth Fund Washington Health Policy Week in Review. June 28, 2010. Available at: http://www.commonwealthfund.org/Content/ Newsletters/Washington-Health-Policy-in-Review/2010/Jun/June-28-2010/ PCORI-Backers-Eye-PR-Strategy.aspx. Accessed September 28, 2011.

11. Chandra A, Jena AB, Skinner JS. The pragmatist's guide to comparative effectiveness research. J Econ Perspect. 2011;25(2):27-46.

12. Vernon JA, Golec JH, Stevens JS. Comparative effectiveness regulations and pharmaceutical innovation. Pharmacoeconomics. 2010;28(10):877-87.

13. Benner JS, Morrison MR, Karnes EK, Kocot SL, McClellan M. An evaluation of recent Federal spending on comparative effectiveness research: priorities, gaps, and next steps. Health Aff (Millwood). 2010;29(10):1768-76. 